\title{
Decoding Strategies at the Relay with Physical-Layer Network Coding
}

\author{
Uttam Bhat and Tolga M. Duman, Fellow, IEEE
}

\begin{abstract}
A two-way relay channel is considered where two users exchange information via a common relay in two transmission phases using physical-layer network coding (PNC). We consider an optimal decoding strategy at the relay to decode the network coded sequence during the first transmission phase, which is approximately implemented using a list decoding (LD) algorithm. The algorithm jointly decodes the codewords transmitted by the two users and sorts the $L$ most likely pair of sequences in the order of decreasing a-posteriori probabilities, based on which, estimates of the most likely network coded sequences and the decoding results are obtained. Using several examples, it is observed that a lower complexity alternative, that jointly decodes the two transmitted codewords, has a performance similar to the LD based decoding and offers a near-optimal performance in terms of the error rates corresponding to the XOR of the two decoded sequences. To analyze the error rate at the relay, an analytical approximation of the word-error rate using the joint decoding (JD) scheme is evaluated over an AWGN channel using an approach that remains valid for the general case of two users adopting different codebooks and using different power levels. We further extend our study to frequency selective channels where two decoding approaches at the relay are investigated, namely; a trellis based joint channel detector/physical-layer network coded sequence decoder (JCD/PNCD) which is shown to offer a near-optimal performance, and a reduced complexity channel detection based on a linear receiver with minimum mean squared error (MMSE) criterion which is particularly useful where the number of channel taps is large.
\end{abstract}

Index Terms-Two-way relay channels, physical-layer network coding, union bounds, joint decoding, list decoding, performance analysis, frequency-selective channel, MMSE detection.

\section{INTRODUCTION}

W E consider a two-way relay channel where two users exchange information via a common relay in two transmission phases. Recently, a scheme referred to as physicallayer network coding (PNC) involving two transmission phases in a two-way relay channel, that incorporates network coding, has been of considerable interest. Network coding was initially proposed in [1] as a means to improve network

Manuscript received February 12, 2012; revised June 4 and July 18, 2012; accepted July 19, 2012. The associate editor coordinating the review of this paper and approving it for publication was W. Zhang.

This work has been presented in part at the IEEE Wireless Communications and Networking Conference (WCNC), Paris, France, April 2012.

This publication was made possible by a NPRP grant from the Qatar National Research Fund (a member of the Qatar Foundation). The statements made herein are solely the responsibility of the authors.

U. Bhat was with the School of Electrical, Computer and Energy Engineering (ECEE), Arizona State University, Tempe, AZ 85287-5706, USA (e-mail: ubhat@asu.edu).

T. Duman is with the Department of Electrical and Electronics Engineering, Bilkent University, Bilkent, Ankara, 06800, Turkey (e-mail: duman@ee.bilkent.edu.tr). He is on leave from the School of ECEE, Arizona State University.

Digital Object Identifier 10.1109/TWC.2012.101112.120190 throughput where the basic idea is that in a communication network, the intermediate nodes route new packets which are a function of the incoming data (e.g. XOR of incoming packets). In the case of network coding at the physical-layer, the relay transforms the linear sum of the signals from the two users during the the first (multiple-access) phase, into a sequence of interpretable symbols before relaying them in the second (broadcast) phase [2]. Several techniques can be adopted at the relay in order to to map the received sequence of superimposed signals into a form that can be decoded at both nodes such as amplify-and-forward [3], successive interference cancellation [4], etc. In [5], a denoise-and-forward based scheme is studied that jointly estimates the pair of modulated symbols transmitted by the two users and maps them to symbols corresponding to discrete constellations based on the channel conditions (that is known at the relay) over the two source-relay links. Here, the focus is on the design of constellation and the mapping to implement the network coding at the relay, where it is shown that for certain channel conditions, incorporation of 5-ary modulation at the relay improves the system performance. An Arithmetic-sum Channel-decoding Network-Coding (ACNC) based decoding scheme is proposed in [4], where it is shown that the decoding performance at the relay can be enhanced by exploiting the dependencies among the symbols created by channel coding. The relay first computes the soft information corresponding to the linear sum of the received sequence of symbols and uses this information to estimate the network coded sequence. Improved performance results with this scheme have been verified in [4] with repeat accumulate codes, and with low density parity check codes in [6]. In the case of PNC with convolutional codes, decoding schemes based on modified maximum likelihood decoding and low complexity reduced state decoding are provided in [7]. Here, a reduced state trellis is constructed from the joint full state trellis by merging the disjoint states into single state (i.e. the XOR of the states corresponding to the different transitions are same). It is shown that the complexity of the reduced state decoding is approximately square root of the full state decoding complexity with no loss of diversity gain for fading channels.

In the case of two-way relay channels in a multi-path propagation environment that exhibit frequency selectivity, the relay has to first combat the inter-symbol interference (ISI) before relaying the network coded signal to each of the destinations during the broadcast phase. This kind of a channel model is typically observed in underwater acoustic (UWA) communications [8] and mobile broadband wireless networks, where the delay spread is very large leading to 
significant frequency-selective signal distortions. Recently, some work has been done in two-way relay channels with frequency selective fading. In [9], an amplify-and-forward scheme is incorporated at the relay, where the relay amplifies the received superimposed signals during the first phase and broadcasts it to the two user nodes during the second phase. Here, a physical-layer network coding using orthogonal frequency division multiplexing (OFDM) and single carrier transmissions with frequency domain equalization (SC-FDE) are investigated using equalization techniques based on zero forcing and MMSE criteria. In [10], a design method based on closest-neighbor clustering is proposed. An adaptive network coding is implemented where based on the channel conditions at the relay, an appropriately designed signal constellation is selected. It is shown that adaptive network coding with with 5ary denoising provides good performance over Rician channels with large decay factors.

In this paper, we start with the optimal decoding strategy at the relay to decode the network coded sequence ${ }^{1}$ from the received superimposed signal. We notice that the implementation of the optimal decoding scheme is formidable; however, it is possible to approximate it by using a list decoding (LD) scheme with a list size large enough to select the most likely codeword pairs corresponding to those transmitted by the two users and then evaluating the likelihood of each bit after marginalization of the sequences in the list. It is shown using extensive numerical examples that the error rate performance with the list decoding scheme which is nearly optimal, is similar to the joint decoding of the pair of codewords transmitted by the two users offering a lower complexity solution. Using the JD scheme, we analyze the system performance where we (approximately) compute the overall worderror rate based on a union bound. Our proposed analytical approximation holds good for finite length coding schemes, including those involving iterative based decoding methods, for different power allocations at each users. Using computer simulations, we provide examples of decoding results and analytical bounds, and show that they are in good agreement with each other. The analysis developed here allows us to study the effectiveness of the finite length coding schemes employed at the two sources. Recently, some work has also been done on performance analysis of physical-layer network coding for two-way relay channels. In [11], performance analysis of two-way relay channels without channel coding is considered when BPSK is employed. Here, the maximum likelihood detection metric at the relay is approximated using the usual max-log approximation, based on which bounds on symbol error rates at the relay are derived. In [12], an error probability bound at the relay is computed using a punctured codebook method. This method is used to compute explicitly the distance spectrum of the physical-layer network coded scheme.

We further investigate decoding strategies for frequency selective two-way relay channels with physical-layer network coding. We focus on single carrier transmissions and start with the optimal decoding scheme which, as in the case of AWGN

\footnotetext{
${ }^{1}$ Here, by a network coded sequence we refer to $\mathbf{s}=\mathbf{s}_{1} \oplus \mathbf{s}_{2}$, where $\mathbf{s}_{i}$ corresponds to the binary codeword generated at the $i^{\text {th }}$ user and $\oplus$ is the binary 'XOR' operator. We note that other encoding options are also possible.
}

channels, is approximately implemented using a list decoding algorithm. It is shown that a joint channel detector/physicallayer network coded sequence decoder, that jointly decodes the pair of transmitted codewords, has a performance similar to the list decoding scheme over frequency selective channels as well. Further, we investigate a minimum mean squared error based detection scheme in order to compute the equalizer weights that can be used to generate soft information of the superimposed signals received at the relay. A PNC decoder then decodes the network coded sequence using the a-priori information from the detector. The MMSE based equalization scheme is a significantly suboptimal approach as also reported for one-way relay channels [13]. However, incorporation of turbo equalization [14], that iteratively exchanges soft information between the MMSE based linear detector and the channel decoder, improves its performance. The MMSE based scheme is particularly useful when the span of the significant channel taps is long for which the implementation of the JCD/PNCD scheme becomes very complex. This work has also been discussed in [15], where the focus is limited to the channels that exhibit frequency selectivity. The case with AWGN channel is not discussed here.

The paper is organized as follows. We describe the system setup in Section II. In Section III, we go over the optimal decoding strategy, motivate the implementation of the list decoding algorithm as an approximation, and give a low complexity joint decoding solution. In Section IV, we derive analytical approximations on the word-error rate using the JD scheme. In Section V, we extend our work to the case of channels with ISI and describe the equivalent channel model. In Section V-A, we investigate the design of detection and decoding schemes where we describe the JCD/PNCD scheme followed by the design of the MMSE scheme for channel detection with a PNC decoder. In Section VI, we provide numerical examples illustrating performance of the different decoding strategies considered and provide comparisons between analytical bounds and simulation results. Finally, we conclude the paper with some remarks in Section VII.

\section{SySTEM DESCRIPTION}

Let $\mathbf{w}_{i}=\left\{w_{i}(1), w_{i}(2), \ldots, w_{i}(K)\right\}$ be the information sequence of length $K$ at the $i^{\text {th }}$ source node encoded to form the binary codeword $\mathbf{s}_{i}=\left\{s_{i}(1), s_{i}(2), \ldots, s_{i}(N)\right\}$ of length $N, i=1,2$. The coded sequence is modulated using binary phase shift keying (BPSK) and is denoted by the length- $N$ sequence $\mathbf{x}_{i}=\left\{x_{i}(1), x_{i}(2), \ldots, x_{i}(N)\right\}$, which is then transmitted over an additive white Gaussian noise (AWGN) channel after assigning power $P_{i}$. We assume that the transmissions are perfectly synchronized and the relay simultaneously receives the messages transmitted by the two users as shown in Fig. 1. An additional interleaver is included at the output of the second modulator, whose significance will become apparent in Section IV. At the relay, the received signal corresponding to the superposition of the $n^{t h}$ transmitted symbols from the two users can be written as

$$
\begin{aligned}
y_{R}(n) & =\sqrt{P_{1}} x_{1}(n)+\sqrt{P_{2}} x_{2}(n)+z_{R}(n), \\
& =x_{R}(n)+z_{R}(n),
\end{aligned}
$$




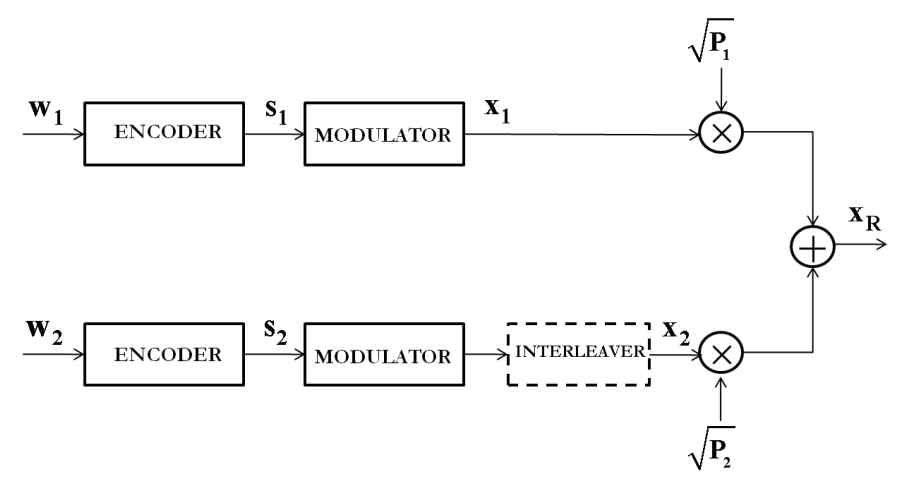

Fig. 1. System setup for a two-way relay channel.

where $\mathbf{z}_{R}=\left\{z_{i}(1), z_{i}(2), \ldots, z_{i}(N)\right\}$ denote a sequence of independent and identically distributed (i.i.d.) Gaussian random variables with zero mean and variance $\sigma^{2}$ and $x_{R}(n) \in\left\{ \pm\left(\sqrt{P_{1}} \pm \sqrt{P_{2}}\right)\right\}$.

We incorporate the PNC strategy at the relay, where the objective is to estimate the information sequence $\mathbf{w}=\mathbf{w}_{\mathbf{1}} \oplus \mathbf{w}_{\mathbf{2}}$ from the received noisy sequence of symbols $\mathbf{y}_{R}$. The decoded sequence is then transmitted simultaneously to both users in the next (broadcast) phase, after encoding and modulation. Here, we focus only on the communication scheme corresponding to the first phase.

\section{Decoding Algorithms at the Relay}

\section{A. Optimal Decoding Scheme}

Let us assume that the two source nodes generate codewords $\mathbf{s}_{1}$ and $\mathbf{s}_{2}$ such that the corresponding network coded sequence is $\mathbf{s}=\mathbf{s}_{1} \oplus \mathbf{s}_{2}$. Also let us define a set $\mathcal{E}(s)$ such that

$$
\mathcal{E}(s)=\left\{\left(\hat{\mathbf{s}}_{1}, \hat{\mathbf{s}}_{2}\right): \hat{\mathbf{s}}_{1} \oplus \hat{\mathbf{s}}_{2}=\mathbf{s}, \hat{\mathbf{s}}_{i} \in \mathcal{C}_{i}, i=1,2\right\},
$$

where $\mathcal{C}_{i}$ denotes the codebook at the $i^{\text {th }}$ user. Assuming that all the codewords for each transmitter are equally likely, the optimal decoding rule for the network coded sequence, $\mathrm{s}$, can then be written as

$$
\begin{aligned}
\hat{\mathbf{s}} & =\arg \max _{\widetilde{\mathbf{s}}} P\left(\tilde{\mathbf{s}} \mid \mathbf{y}_{R}\right) \\
& =\arg \max _{\widetilde{\mathbf{s}}} \sum_{\left(\hat{\mathbf{s}}_{1}, \hat{\mathbf{s}}_{2}\right) \in \mathcal{E}(\tilde{s})} P\left(\hat{\mathbf{s}}_{1}, \hat{\mathbf{s}}_{2} \mid \mathbf{y}_{R}\right) \\
& =\arg \max _{\widetilde{\mathbf{s}}} \sum_{\left(\hat{\mathbf{s}}_{1}, \hat{\mathbf{s}}_{2}\right) \in \mathcal{E}(\tilde{s})} \frac{P\left(\mathbf{y}_{R} \mid \hat{\mathbf{s}}_{1}, \hat{\mathbf{s}}_{2}\right) P\left(\hat{\mathbf{s}}_{1}, \hat{\mathbf{s}}_{2}\right)}{P\left(\mathbf{y}_{R}\right)} \\
& =\arg \max _{\widetilde{\mathbf{s}}} \sum_{\left(\hat{\mathbf{s}}_{1}, \hat{\mathbf{s}}_{2}\right) \in \mathcal{E}(\tilde{s})} \exp \left(\frac{-\left\|\mathbf{y}_{R}-\sqrt{P_{1}} \hat{\mathbf{x}}_{1}-\sqrt{P_{2}} \hat{\mathbf{x}}_{2}\right\|^{2}}{2 \sigma^{2}}\right) \cdot(
\end{aligned}
$$

We observe that the exact implementation of the optimal decoding scheme in (3) is not feasible since the set $\mathcal{E}(s)$ contains a large number of elements (exponential in codeword length). Therefore, we next resort to alternative schemes that can be used as approximations.

\section{B. A List Decoding Based Scheme}

A good approximation to the decoding rule in (3) can be developed by implementing a list decoding algorithm that makes use of a metric based on minimum squared Euclidean distance criterion. A list decoding scheme (with a list size $L$ ) selects the $L$ most likely pairs of sequences corresponding to the codewords transmitted by the two users and sorts them in the order of decreasing a-posteriori probabilities. Hence, the dominant terms of the summation in (3) can be identified. Using the list of $L$ most likely codeword pairs, the objective is then to find the most likely sequence by evaluating the likelihood of each bit after marginalization of the $L$ sequences in the list. We note that the approach adopted here is similar to a MAP decoding scheme where the resulting sequence obtained in this manner is not necessarily a (network) coded sequence. The idea of list decoding applied to the physicallayer network coding is general; that is, different types of codes at the individual nodes can be employed. Here, we focus on the use of convolutional and turbo codes. An extended trellis for a joint encoder with generators corresponding to the codebooks employed at the two users is constructed to implement these codes. If the encoder at the $i^{t h}$ user is represented using a trellis with $2^{S_{i}}$ states and $2^{M_{i}}$ inputs, then the new trellis has effectively $2^{S_{1}+S_{2}}$ states and $2^{M_{1}+M_{2}}$ inputs.

1) List Decoding of Convolutional Codes: In order to decode the received sequence at the relay, we make use of the Viterbi algorithm using the extended state representation. The metric corresponding to the $n$th received symbol for each state transition can be computed as

$$
\mathcal{M}_{\left(s, s^{\prime}\right)}^{(n)}=\left|y^{s}(n)-x^{s}\right|^{2}+\left|y^{p}(n)-x^{p}\right|^{2},
$$

where $\left(\mathbf{y}^{s}, \mathbf{y}^{p}\right)$ are the noisy sequences of systematic and parity symbols received at the relay and

$$
\begin{aligned}
& x^{s}=\sqrt{P_{1}}\left(2 i_{1}-1\right)+\sqrt{P_{2}}\left(2 i_{2}-1\right), \\
& x^{p}=\sqrt{P_{1}}\left(2 p_{1}-1\right)+\sqrt{P_{2}}\left(2 p_{2}-1\right),
\end{aligned}
$$

where $\left(i_{j}, p_{j}\right)$ are the information and parity bits of the $j^{\text {th }}$ user corresponding to the transition from state $s$ to state $s^{\prime}$. To generate the list of most likely pairs of codewords, various specific list decoding algorithms can be implemented including the serial list Viterbi Algorithm (SLVA), parallel LVA (PLVA) [16] and improved SLVA [17].

2) List Decoding of Turbo Codes: We extend our study of the decoding scheme with iteratively decodable codes such as the turbo codes. In our setup, we assume that both users employ the same interleavers during the encoding process so that turbo decoding can be implemented with the BCJR algorithm using the extended trellis (other approaches that incorporate different interleavers at the users are possible). Turbo decoding is carried out in $M$ iterations, where during each iteration, extrinsic information is exchanged between the two inner MAP decoders. At the end of the $M$ th iteration, the extrinsic information is passed to a list Viterbi decoder, which then generates a list of $L$ most likely sequences in a decreasing order of their probabilities [18]. The extended trellis is incorporated during both, the turbo decoding as well as the list Viterbi decoding stage. The branch metric for the transition from state $s$ to state $s^{\prime}$ corresponding to the input pair $\left(i_{1}, i_{2}\right)$ during list decoding is given by (5) at the top of the next page, where $L_{\left(i_{1}, i_{2}\right)}^{e x t}$ is the a-priori information from the second MAP decoder at the end of the $M^{t h}$ iteration of turbo decoding. 


$$
\mathcal{M}_{\left(s, s^{\prime}\right)}^{(n)}=-L_{\left(i_{1}, i_{2}\right)}^{e x t}+\left|y^{s}(n)-\left(\sqrt{P_{1}}\left(2 i_{1}-1\right)+\sqrt{P_{2}}\left(2 i_{2}-1\right)\right)\right|^{2}+\left|y^{p}(n)-\left(\sqrt{P_{1}}\left(2 p_{1}-1\right)+\sqrt{P_{2}}\left(2 p_{2}-1\right)\right)\right|^{2} .
$$

\section{A Simplified Decoding Strategy - Joint Decoding}

We observe that at high signal-to-noise ratios (SNRs), i.e., $\sigma^{2} \rightarrow 0$, only one of the terms in (3) is dominant, for which the equivalent decoding rule may be approximated as

$$
\hat{\mathbf{s}}=\arg \max _{\tilde{\mathbf{s}}=\tilde{\mathbf{s}}_{1} \oplus \tilde{\mathbf{s}}_{2}} \exp \left(-\frac{\| \mathbf{y} R}{-\sqrt{P_{1}} \tilde{\mathbf{x}}_{1}-\sqrt{P_{2}} \tilde{\mathbf{x}}_{2} \|^{2}} 2^{2}\right) .
$$

From this high SNR approximation, we define a joint decoding scheme at the relay for the two codewords as

$$
\left(\mathbf{x}_{1}^{(J D)}, \mathbf{x}_{2}^{(J D)}\right)=\arg \min _{\left(\tilde{\mathbf{s}}_{1}, \tilde{\mathbf{s}}_{2}\right)}\left\|\mathbf{y}-\sqrt{P_{1}} \tilde{\mathbf{x}}_{\mathbf{1}}-\sqrt{P_{2}} \tilde{\mathbf{x}}_{\mathbf{2}}\right\|^{2}
$$

where $\mathbf{x}_{1}^{(J D)}$ and $\mathbf{x}_{2}^{(J D)}$ are the decisions made by the relay corresponding to the first and the second messages respectively, and the minimization is performed over the codebooks of both the messages. The equivalent network coded sequence decoded at the relay is then given by

$$
\mathbf{s}^{(J D)}=\mathbf{s}_{1}^{(J D)} \oplus \mathbf{s}_{2}^{(J D)} .
$$

We note that if the relay were to decode $\mathbf{x}_{\mathbf{1}}{ }^{(J D)}$ and $\mathbf{x}_{\mathbf{2}}{ }^{(J D)}$ only, and not the network coded sequence $\mathbf{s}^{(J D)}$, then the strategy described in (7) would be the optimal decoding scheme, and hence would have provided the optimal error-rate performance. However, since we are interested in decoding of the $X O R$ of the transmitted codewords, the JD scheme is a sub-optimal approach with near-optimum performance as will be assessed using extensive numerical examples.

\section{ANALysis of ERror Probability}

We now proceed with the evaluation of the performance bounds of the codes employed at the two users for the JD scheme. We assume that the codes at each of the user nodes are linear, consisting of independent and uniformly distributed bits, and that their distance spectra are known. Namely, the number $t_{1}(d)$ (respectively, $t_{2}(d)$ ) of codewords with Hamming weight $d$ in the first (respectively, the second) codebook is assumed to be known, for each value of $d$ in $\{1,2, \ldots, N\}$. In the case of turbo-like codes involving interleavers, the distance spectra $t_{1}(d)$ and $t_{2}(d)$ are intended as statistical averages with uniform interleaving [19].

Assuming that $\mathbf{x}_{1}$ and $\mathbf{x}_{2}$ are transmitted by each of the sources, the conditional error probability corresponding to $\mathbf{s}=$ $\mathbf{s}_{1} \oplus \mathbf{s}_{2}$ with the JD scheme can be written as

$$
P_{\mathrm{e}}^{(\mathrm{JD})}(s)=P\left(\mathbf{s}^{(J D)} \neq \mathbf{s} \mid \mathbf{s}\right) .
$$

Since the exact evaluation of the error probability $P_{\mathrm{e}}^{(\mathrm{JD})}$ is not practically feasible for most codes of interest, we evaluate the union bound for $P_{\mathrm{e}}^{(\mathrm{JD})}$ for which we can rewrite the expected value of (8) as

$$
\begin{aligned}
P_{\mathrm{e}}^{(\mathrm{JD})} & =\mathbb{E}\left[P\left(\mathbf{s}^{(J D)} \neq \mathbf{s} \mid \mathbf{x}_{\mathbf{1}}, \mathbf{x}_{\mathbf{2}}\right)\right], \\
& \leq \mathbb{E}\left[P\left(\left(\mathbf{x}_{\mathbf{1}}^{(J D)}, \mathbf{x}_{\mathbf{2}}^{(J D)}\right) \neq\left(\mathbf{x}_{1}, \mathbf{x}_{2}\right) \mid \mathbf{x}_{\mathbf{1}}, \mathbf{x}_{\mathbf{2}}\right)\right],
\end{aligned}
$$

where $\mathbb{E}[\cdot]$ is the statistical expectation over all the codewords of the two users (and over all interleavers). The inequality follows, since with the incorporation of the PNC scheme, $\mathbf{s}^{(J D)}$ may be correctly decoded even when $\mathbf{x}_{\mathbf{1}}{ }^{(J D)}$ and $\mathbf{x}_{\mathbf{2}}{ }^{(J D)}$ are in error as there could be more than one pair of codewords that result in the same network coded sequence.

The term $P\left(\left(\mathbf{x}_{\mathbf{1}}{ }^{(J D)}, \mathbf{x}_{\mathbf{2}}{ }^{(J D)}\right) \neq\left(\mathbf{x}_{1}, \mathbf{x}_{2}\right) \mid \mathbf{x}_{\mathbf{1}}, \mathbf{x}_{\mathbf{2}}\right)$ in (9) can be upper bounded using the union bound

$$
\begin{gathered}
P\left(\left(\mathbf{x}_{\mathbf{1}}{ }^{(J D)}, \mathbf{x}_{\mathbf{2}}^{(J D)}\right) \neq\left(\mathbf{x}_{1}, \mathbf{x}_{2}\right) \mid \mathbf{x}_{\mathbf{1}}, \mathbf{x}_{\mathbf{2}}\right) \\
\quad \leq \sum_{\left(\hat{\mathbf{s}}_{1}, \hat{\mathbf{s}}_{2}\right) \neq\left(\mathbf{s}_{1}, \mathbf{s}_{2}\right)} P\left(\left(\mathbf{x}_{\mathbf{1}}, \mathbf{x}_{\mathbf{2}}\right) \rightarrow\left(\hat{\mathbf{x}}_{1}, \hat{\mathbf{x}}_{2}\right)\right),
\end{gathered}
$$

where $P\left(\left(\mathbf{x}_{1}, \mathbf{x}_{2}\right) \rightarrow\left(\hat{\mathbf{x}}_{1}, \hat{\mathbf{x}}_{2}\right)\right)$ is the pairwise error probability of receiving a signal closer to an incorrect network coded sequence $\hat{\mathbf{s}}=\hat{\mathbf{s}}_{1} \oplus \hat{\mathbf{s}}_{2}$ when the codewords, $\mathbf{s}_{1}$ and $\mathbf{s}_{2}$ are transmitted by the first and the second users, respectively. Each term inside the summation is equivalent to (11) at the top of the next page, which after few manipulations can be shown to be

$P\left(P_{1} d_{1}+P_{2} d_{2}+2 \sqrt{P_{1} P_{2}} \sum_{v} \bar{x}_{1, v} \bar{x}_{2, v}+Z \leq 0 \mid \mathbf{x}_{\mathbf{1}}, \mathbf{x}_{\mathbf{2}}\right)$,

where $d_{1}=d_{H}\left(\hat{\mathbf{x}}_{1}, \mathbf{x}_{\mathbf{1}}\right)$ and $d_{2}=d_{H}\left(\hat{\mathbf{x}}_{\mathbf{2}}, \mathbf{x}_{\mathbf{2}}\right)$ are the number of bit errors in $\hat{\mathbf{x}}_{1}$ and $\hat{\mathbf{x}}_{2}$, respectively, $\bar{x}_{i, k}=\frac{x_{i, k}-\hat{x}_{i, k}}{2}$ and $Z$ is a Gaussian random variable whose parameters shall be derived later. Given the values of $d_{1}$ and $d_{2}$, we need the distribution of the random variable $V$, that is, the probability that two sequences with $d_{1}$ and $d_{2}$ errors have $V$ positions where the errors overlap. In general, the conditional distribution $P\left(V \mid d_{1}, d_{2}\right)$ depends on the geometry of the codes employed at the two users. The introduction of the uniform interleaver at the output of the second encoder removes the dependence on the geometry and the problem becomes purely combinatorial. The results in Section VI show that when turbo-like codes are considered, this approximation is very accurate since the output of turbo encoders typically resemble independent and uniformly distributed bits. The term in (12) does not contribute to the error bound when $d_{1}=d_{2}$ and $V=d_{1}$, which means that the positions of the bit errors in both the codewords $\hat{\mathbf{x}}_{1}$ and $\hat{\mathbf{x}}_{2}$ are exactly the same and $\hat{\mathbf{s}}_{\mathbf{1}} \oplus \hat{\mathbf{s}}_{\mathbf{2}}$ results in s. Hence, following the derivation in [20], [21], we see that the random variable $V$ takes values on $\{0,1, \ldots, d\}$ with conditional probability mass function

$$
p_{V}\left(v \mid d_{1}, d_{2}\right)=\frac{\left(\begin{array}{c}
\min \left(d_{1}, d_{2}\right) \\
v
\end{array}\right)\left(\begin{array}{c}
N-\min \left(d_{1}, d_{2}\right) \\
\max \left(d_{1}, d_{2}\right)-v
\end{array}\right)}{N},
$$

where

$$
d= \begin{cases}\min \left(d_{1}, d_{2}\right) & \text { if } d_{1} \neq d_{2} \\ d_{1}-1 & \text { if } d_{1}=d_{2}\end{cases}
$$

Using the decoding strategy given in (7), and after a few manipulations similar to the ones described in [21], each term inside the summation in (10) can be equivalently written as 


$$
\begin{gathered}
P\left(\left\|\mathbf{y}-\sqrt{P_{1}} \hat{\mathbf{x}}_{\mathbf{1}}-\sqrt{P_{2}} \hat{\mathbf{x}}_{\mathbf{2}}\right\|^{2} \leq\left\|\mathbf{y}-\sqrt{P_{1}} \mathbf{x}_{\mathbf{1}}-\sqrt{P_{2}} \mathbf{x}_{\mathbf{2}}\right\|^{2} \mid \mathbf{x}_{\mathbf{1}}, \mathbf{x}_{\mathbf{2}}\right) \\
\sum_{v=0}^{d} \sum_{g=0}^{v} \mathbb{P}\left(P_{1} d_{1}+P_{2} d_{2}+2 \sqrt{P_{1} P_{2}}(v-g)+Z \leq 0\right) p_{V}\left(v \mid d_{1}, d_{2}\right) p_{G}(g \mid V=v) .
\end{gathered}
$$

shown in (13), where, for a given $V$, we define a random variable $G$ as the number of positions where $\left\{v_{n}\right\}$ takes on the value -1 (with +1 at $V-G$ positions). The random variable $G$ takes values on $\{0,1, \ldots, V\}$ with binomial distribution

$$
p_{G}(g \mid V=v)=\frac{1}{2^{v}}\left(\begin{array}{l}
v \\
g
\end{array}\right)
$$

based on the approximation that the employed codebooks are such that the random variables $\left\{v_{n}\right\}$ with $v_{n}=\bar{x}_{1, n} \bar{x}_{2, n}$ are independent and uniformly distributed on $\{-1,+1\}$. Conditioned on $V=v$ and $G=g, Z$ is a Gaussian random variable with zero mean and variance

$\left(P_{1} d_{1}+P_{2} d_{2}+2 \sqrt{P_{1} P_{2}}(v-2 g)\right) \sigma^{2}$.

Therefore, the union bound in (9) can be written as

$$
P_{\mathrm{e}}^{(\mathrm{JD})} \leq \mathbb{E}\left[\sum_{\hat{\mathbf{s}} \neq \mathbf{s}} Q\left(\sqrt{\gamma\left(d_{1}, d_{2}, v, g\right)}\right) \mid \mathbf{x}_{\mathbf{1}}, \mathbf{x}_{\mathbf{2}}\right],
$$

where

$$
\gamma\left(d_{1}, d_{2}, v, g\right)=\left(P_{1} d_{1}+P_{2} d_{2}+2 \sqrt{P_{1} P_{2}}(v-2 g)\right) / \sigma^{2},
$$

and $Q(\cdot)$ is the standard Q-function.

To manage the expectation in (14), we rely on the assumption that both codes are linear, which assures that any possible value of $d_{1}$ (respectively, $d_{2}$ ) equals the Hamming weight of a valid codeword in the first (respectively, second) codebook. Hence, we can rewrite the union bound (14) as shown in (15) at the top of the next page.

\section{Extension to ChanNELS With ISI}

In this section, we shall study the decoding strategies for two-way relay channels in a multipath environment that exhibit inter-symbol interference. The discrete time mathematical model for the received superimposed signal at the relay during the $n^{\text {th }}$ time instant is given by

$$
\begin{aligned}
y_{R}(n)= & \sum_{k=0}^{L_{1}-1} \sqrt{P_{1}} h_{1}(k) x_{1}(n-k) \\
& +\sum_{k=0}^{L_{2}-1} \sqrt{P_{2}} h_{2}(k) x_{2}(n-k)+z_{R}(n),
\end{aligned}
$$

where $\mathbf{z}_{R}=\left\{z_{R}(1), z_{R}(2), \ldots, z_{R}(N)\right\}$ denotes a sequence of length $N$ i.i.d. circularly symmetric complex Gaussian random variables with zero mean and variance $\sigma^{2} / 2$ per dimension, and $\mathbf{h}_{i}=\left\{h_{i}(0), h_{i}(1), \ldots, h_{i}\left(L_{i}-1\right)\right\}$ is the complex channel gain vector of length $L_{i}, i=1,2$, over the link between the $i^{t h}$ user and the relay. We assume that the channel is constant over the transmission of each frame of data and that the channel gains are known at the relay. The objective of the decoder at the relay is to estimate the binary information sequence $\mathbf{w}=\mathbf{w}_{\mathbf{1}} \oplus \mathbf{w}_{\mathbf{2}}$ from the received noisy sequence of symbols $\mathbf{y}_{R}=\left\{y_{R}(1), y_{R}(2), \ldots, y_{R}(N)\right\}$ in the presence of inter-symbol interference.

\section{A. Decoding Schemes at the Relay}

Without loss of generality, we assume that the number of channel taps over the two source-relay links are the same, i.e., $L_{1}=L_{2}=L_{R}$. Also, the channel is assumed to be known at the relay and is constant over the entire length of the codewords. Two different decoding strategies are investigated, namely; a trellis based joint channel detector/physical-layer network coded sequence decoder, and a reduced complexity channel detection based on a linear receiver with MMSE criterion followed by the PNC decoder. Let us assume that the two source nodes generate codewords $\mathbf{s}_{1}$ and $\mathbf{s}_{2}$ such that the corresponding network coded sequence is $\mathbf{s}=\mathbf{s}_{1} \oplus \mathbf{s}_{2}$. Also let us define a set $\mathcal{E}(s)$ such that

$$
\mathcal{E}(s)=\left\{\left(\hat{\mathbf{s}}_{1}, \hat{\mathbf{s}}_{2}\right): \mathbf{s}=\hat{\mathbf{s}}_{1} \oplus \hat{\mathbf{s}}_{2}, \hat{\mathbf{s}}_{i} \in \mathcal{C}_{i}, i=1,2\right\},
$$

where $\mathcal{C}_{i}$ denotes the codebook at the $i^{\text {th }}$ user and $\hat{\mathbf{x}}_{\mathbf{i}}$ is the BPSK modulated sequence corresponding to $\hat{\mathbf{s}}_{\mathbf{i}}$. It is easy to see that the maximum likelihood based decoding rule can be developed for frequency selective channels with known channel state information at the relay, which on following steps similar to (3), may be written as

$$
\hat{\mathbf{s}}=\arg \max _{\tilde{\mathbf{s}}} \sum_{\left(\hat{\mathbf{s}}_{1}, \hat{\mathbf{s}}_{2}\right) \in \mathcal{E}(\tilde{s})} \exp \left(\frac{-\left\|\mathbf{d}_{I S I}\right\|^{2}}{\sigma^{2}}\right),
$$

where $\mathbf{d}_{I S I}=\mathbf{y}_{R}-\left(\sqrt{P_{1}}\left(\mathbf{h}_{1} \star \hat{\mathbf{x}}_{1}\right)+\sqrt{P_{2}}\left(\mathbf{h}_{2} \star \hat{\mathbf{x}}_{2}\right)\right)$ and $\star$ denotes linear convolution of two vectors. We note that as the length of the codewords increase, the implementation of this strategy becomes infeasible and other lower complexity techniques are warranted. Hence, we resort to a list decoding algorithm that can be employed to approximate the optimal decoding strategy.

1) List Decoding Scheme: We implement the optimal decoding strategy approximately using a list decoding scheme with a list size $L$, that incorporates a metric based on minimum Euclidean distance criterion. From (17), it is observed that the network coded sequence $\mathrm{s}$ can be formed by more than one pair of codewords corresponding to the two users. Using the LD scheme allows us to identify the pair of codewords from the set $\mathcal{E}(s)$, that dominate the summation in (18). The objective with the list decoding algorithm is then to find the most likely network coded sequence from the list after marginalization of the $L$ sequences identified.

When convolutional codes are employed at the two user nodes, a list Viterbi algorithm using parallel list Viterbi algorithm [16], [22] is implemented ${ }^{2}$. In order to implement

\footnotetext{
${ }^{2}$ We note that other decoding algorithms such as SLVA [16] and improved SLVA [17] can also be applied.
} 


$$
P_{\mathrm{e}}^{(\mathrm{JD})} \leq \sum_{\left(d_{1}, d_{2}\right) \neq(0,0)} \sum_{v=0}^{d} \sum_{g=0}^{v} t_{1}\left(d_{1}\right) t_{2}\left(d_{2}\right) p_{V}\left(v \mid d_{1}, d_{2}\right) p_{G}(g \mid v) Q\left(\sqrt{\gamma\left(d_{1}, d_{2}, v, g\right)}\right)
$$

this scheme, we construct a joint trellis using the trellis of the convolutional code and the trellis representing the channel state corresponding to each user. Using the joint trellis of each of the users, an extended trellis is constructed, the state representation of which is used in the implementation of the list decoding algorithm. As an example, the total number of states in an extended trellis using a 4-state convolutional code at each node with a 2-tap ISI channel turns out to be 64 . The complexity of the decoder at the relay increases exponentially with the length of the channel taps. For instance, when the number of taps in the ISI channel increases to 3 , the total number of states in the extended trellis is 256 . Hence, lower complexity suboptimal schemes involving reduced number of states, such as the M-algorithm [23] or the T-algorithm [24], can be incorporated at the relay to decode the network coded sequence as a more efficient solution.

2) Joint Channel Detector/Physical-Layer Network Coded Sequence Decoder: At high SNRs, the received sequence of superimposed signals would be closer to one of the pairs of codewords (in the Euclidean distance sense) in the set $\mathcal{E}(s)$. Using this high SNR approximation, a joint decoding rule at the relay can be written as

$$
\left(\mathbf{x}_{1}^{(J D)}, \mathbf{x}_{2}^{(J D)}\right)=\arg \min _{\left(\tilde{\mathbf{s}}_{\mathbf{1}}, \tilde{\mathbf{s}}_{\mathbf{2}}\right)}\left\|\tilde{\mathbf{d}}_{I S I}\right\|^{2}
$$

where $\mathbf{x}_{1}^{(J D)}$ and $\mathbf{x}_{2}^{(J D)}$ are the decisions made by the relay corresponding to the first and the second messages respectively, $\tilde{\mathbf{d}}_{I S I}=\mathbf{y}_{R}-\left(\sqrt{P_{1}}\left(\mathbf{h}_{1} \star \tilde{\mathbf{x}}_{1}\right)+\sqrt{P_{2}}\left(\mathbf{h}_{2} \star \tilde{\mathbf{x}}_{2}\right)\right)$ and the minimization is performed over the codebooks of both the messages. As in the case of channels with no ISI, the JCD/PNCD offers a sub-optimal solution; however, our numerical investigations show that it provides a very good performance comparable to the list decoding solution ${ }^{3}$. Once the two codewords are decoded, the equivalent network coded sequence at the relay can be obtained by

$$
\mathbf{s}^{(J D)}=\mathbf{s}_{1}^{(J D)} \oplus \mathbf{s}_{2}^{(J D)} .
$$

3) Reduced Complexity Linear Detection: The trellis based decoding algorithm discussed earlier provides a near-optimal performance but the complexity of the JCD/PNCD increases exponentially with the number of the channel taps. Even though we can resort to other lower complexity suboptimal trellis based schemes (such as the $M$-algorithm), it is essential to develop other low-complexity detection algorithms to provide a good trade-off between complexity and performance. Hence, we consider a minimum mean squared error based linear detector followed by a PNC decoder at the relay instead of a joint detector/decoder. The MMSE equalizer generates soft information corresponding to the linear sum of the symbols transmitted by the two users, and passes it to the decoder

\footnotetext{
${ }^{3}$ The error rate performance with LD scheme (which is an approximate implementation of the optimal decoding strategy) is similar to the JCD/PNCD scheme.
}

(which in our case is a Viterbi decoder). Before we proceed, we define the following terms

$$
\begin{aligned}
\mathbf{y}_{n} & =\left[y\left(n-K_{2}\right) y\left(n-K_{2}+1\right) \cdots y\left(n+K_{1}\right)\right]^{T}, \\
\mathbf{x}_{i, n} & =\left[x_{i}\left(n-K_{2}-L_{R}+1\right) \cdots x_{i}\left(n+K_{1}\right)\right]^{T}, \\
\mathbf{z}_{n} & =\left[z\left(n-K_{2}\right) z\left(n-K_{2}+1\right) \cdots z\left(n+K_{1}\right)\right]^{T},
\end{aligned}
$$

where, $\mathbf{x}_{i, n}$ represents the sequence of transmitted symbols from $i^{t h}$ user, $\mathbf{y}_{n}$ is the sequence of observations at the relay and $\mathbf{z}_{n}$ corresponds to the noise sequence, $K_{1}$ and $K_{2}$ are the lengths of the noncausal and causal part of the estimator filter respectively, and $K_{R}=K_{1}+K_{2}+1$ is the overall filter length observed at the relay. The objective of the detector is to evaluate an estimate, $\hat{x}(n)$, of $x(n)=\sqrt{P_{1}} x_{1}(n)+\sqrt{P_{2}} x_{2}(n)$.

The channel model can be equivalently written as

$$
\mathbf{y}_{n}=\sqrt{P_{1}} \mathbf{H}_{1} \mathbf{x}_{1, n}+\sqrt{P_{2}} \mathbf{H}_{2} \mathbf{x}_{2, n}+\mathbf{z}_{n},
$$

where the channel convolution matrix, with a dimension $K_{R} \times$ $\left(K_{R}+L_{R}-1\right)$, corresponding to the $i^{t h}$ link is as shown in (21) at the top of the next page The estimate of the signal $x(n)$ can be formed as

$$
\hat{x}(n)=\mathbf{c}_{n}^{H} \mathbf{y}_{n},
$$

where $\mathbf{c}_{n}=\left[c_{n}\left(-K_{2}\right) c_{n}\left(-K_{2}+1\right) \ldots c_{n}(0) \ldots c_{n}\left(K_{1}\right)\right]^{T}$ and $\hat{x}(n)=\sqrt{P_{1}} \hat{x}_{1}(n)+\sqrt{P_{2}} \hat{x}_{2}(n)$. In order to compute optimal equalizer coefficients, the function that is to be minimized with respect to $\mathbf{c}_{n}$ is expressed as

$$
e(n)=\mathbb{E}\left[\left|\mathbf{c}_{n}^{H} \mathbf{y}_{n}-x(n)\right|^{2}\right]
$$

After some straightforward computations, it can be shown that

$$
\mathbf{c}_{n}=\operatorname{Cov}\left(\mathbf{y}_{n}, \mathbf{y}_{n}\right)^{-1} \operatorname{Cov}\left(\mathbf{y}_{n}, x(n)\right),
$$

where

$$
\begin{aligned}
\operatorname{Cov}\left(\mathbf{y}_{n}, \mathbf{y}_{n}\right) & =P_{1} \mathbf{H}_{1} \mathbf{H}_{1}^{H}+P_{2} \mathbf{H}_{2} \mathbf{H}_{2}^{H}+\sigma^{2} I_{N}, \\
\operatorname{Cov}\left(\mathbf{y}_{n}, x(n)\right) & =P_{1} \mathbf{s}_{1}+P_{2} \mathbf{s}_{2},
\end{aligned}
$$

with $\mathbf{s}_{i}=\mathbf{H}_{i}\left[\mathbf{0}_{1 \times\left(K_{2}+L_{R}-1\right)} 1 \mathbf{0}_{1 \times K_{1}}\right]^{T}$ and $\operatorname{Cov}(\mathbf{a}, \mathbf{b})=$ $\mathbb{E}\left[(\mathbf{a}-\mathbb{E}[\mathbf{a}])(\mathbf{b}-\mathbb{E}[\mathbf{b}])^{H}\right]$.

In order to derive a soft-output detector, we treat $\hat{x}(n)$ as a random variable with Gaussian distribution (conditioned on the superposition of correct symbols). Then the conditional probability density function $P(\hat{x}(n) \mid x(n))$ can be computed using the first and second order averages $\mu_{x}(n)$ and $\sigma_{x}^{2}(n)$, which are, respectively, the mean and the variance of the estimated value, $\hat{x}(n)$, conditioned on $x(n)$. Since the transmitted symbols at each node are BPSK modulated, the estimated symbol will resemble a 4-level pulse amplitude modulation scheme. The conditional mean of $\hat{x}(n)$ is computed as

$$
\begin{aligned}
\mu_{x}(n) & =\mathbb{E}[\hat{x}(n) \mid x(n)=x] \\
& =\mathbf{c}_{n}^{H}\left(\sqrt{P_{1}} x_{1} \mathbf{s}_{1}+\sqrt{P_{2}} x_{2} \mathbf{s}_{2}\right) .
\end{aligned}
$$




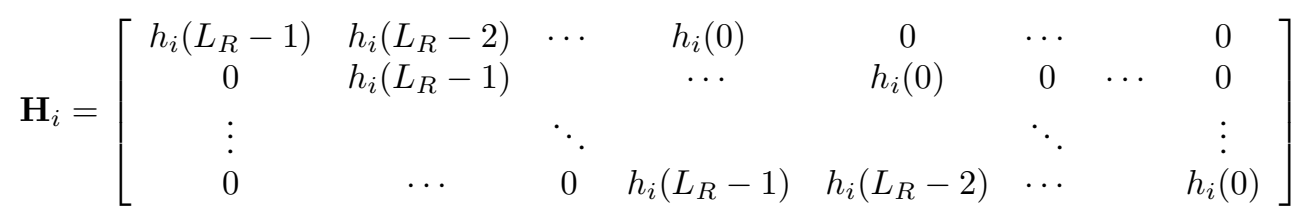

The conditional variance, $\sigma_{x}^{2}(n)$, of $\hat{x}(n)$ can be computed as

$$
\begin{aligned}
\sigma_{x}^{2}(n) & =\mathbb{E}\left[\left|\mathbf{c}_{n}^{H} \mathbf{y}_{n}-\mu_{x}(n)\right|^{2} \mid x(n)=x\right], \\
& =\mathbf{c}_{n}^{H} \mathbb{E}\left[\mathbf{y}_{n} \mathbf{y}_{n}^{H} \mid x(n)=x\right] \mathbf{c}_{n}-\left|\left(\mu_{x}(n)\right)\right|^{2} .(24)
\end{aligned}
$$

After further algebra, we obtain

$$
\begin{aligned}
\mathbb{E}\left[\mathbf{y}_{n} \mathbf{y}_{n}^{H} \mid x(n)=x\right] & =\operatorname{Cov}\left(\mathbf{y}_{n}, \mathbf{y}_{n}\right)+J, \\
\left|\mu_{x}(n)\right|^{2} & =\mathbf{c}_{n}^{H}\left(P_{1} S_{1}+P_{2} S_{2}+J\right) \mathbf{c}_{n},
\end{aligned}
$$

where $J=\sqrt{P_{1} P_{2}} x_{1} x_{2}\left(\mathbf{s}_{1} \mathbf{s}_{2}^{H}+\mathbf{s}_{2} \mathbf{s}_{1}^{H}\right)$ and $S_{i}=\mathbf{s}_{i} \mathbf{s}_{i}^{H}$, from which we can find

$$
\sigma_{x}^{2}(n)=\mathbf{c}_{n}^{H}\left(\operatorname{Cov}\left(\mathbf{y}_{n}, \mathbf{y}_{n}\right)-P_{1} S_{1}-P_{2} S_{2}\right) \mathbf{c}_{n} .
$$

The soft information computed using the mean and the variance computed in (23) and (26), respectively, is then passed to a Viterbi decoder, where for each state transition, the metric corresponding to the $n^{t h}$ symbol is evaluated. The transmitted codewords are then jointly decoded using the computed metric.

The performance of the system can be further improved by incorporating an iterative detection/decoding scheme, i.e., turbo equalization [14] where soft information is exchanged between the MMSE equalizer and the Viterbi decoder in an iterative manner. In this paper, we focus only on the single stage detection/decoding scheme.

\section{NUMERICAL EXAMPLES}

In this section, we provide several results obtained using computer simulations for the optimal decoding scheme in (3) approximated using the list decoding algorithm described in Section III-B, and also compare the bounds obtained using the analytical expression and the simulation results with the joint decoding strategy. We consider a list Viterbi decoder implemented using PLVA with a list size of $L=100$ and we define the SNR as $\left(P_{1}+P_{2}\right) / \sigma^{2}$. In Fig. 2 , rate- $1 / 2$ convolutional codes are employed at both nodes with same constituent encoders $(5 / 7)_{\text {octal }}$ where VD implies JD scheme implemented using Viterbi decoding. We observe that with the JD scheme, the loss in the performance is just 0.015 $\mathrm{dB}$ compared to the $\mathrm{LD}$ scheme in order to achieve a bit error rate (BER) of $10^{-2}$. Fig. 3 shows the performance of LD and JD schemes when turbo codes are employed. Here, the users employ different constituent convolutional codes $\left((5 / 7)_{\text {octal }}\right.$ and $\left.(7 / 5)_{\text {octal }}\right)$ but with same power allocation (and same interleavers) at both the nodes. In the case of list decoding, the turbo decoder performs $M=15$ iterations at the end of which the soft values corresponding to each of the $K$ information bits are passed to a list Viterbi decoder. From the simulation results, we observe a similar behavior, i.e., the performance of the JD scheme is very close to that of the LD scheme. In general, for a given total power $P\left(P=P_{1}+P_{2}\right)$, the best performance at the relay is

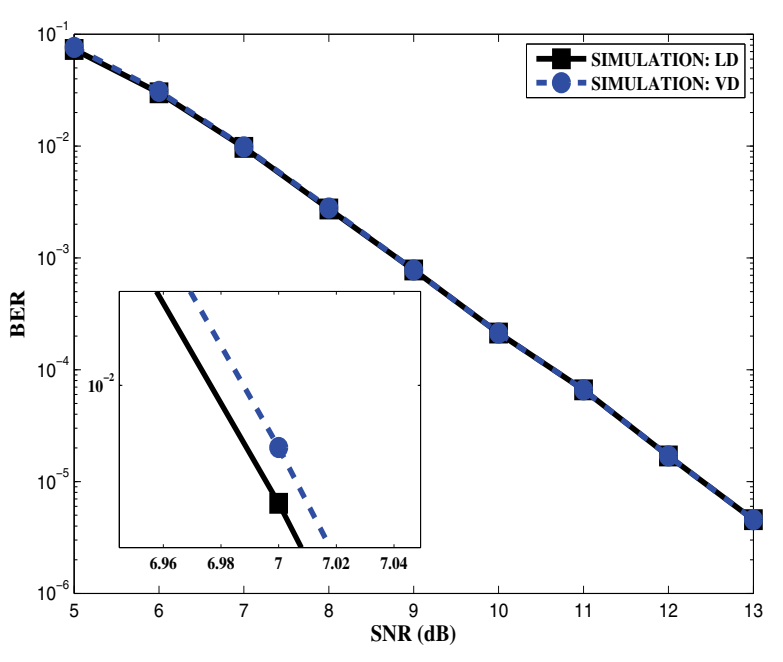

Fig. 2. Simulation results with same $(5 / 7)_{\text {octal }}$ convolutional codes at the two source nodes with $N=256, P_{1}=1$ and $P_{2}=0.8$.

observed when both users employ same power allocation, i.e. $P_{1}=P_{2}$. In this setup, the superimposed symbol $x_{R}(n)$ takes on one of the three constellation points as opposed to four when the powers are different. For the same power $P$, the three constellation scheme has higher minimum distance and is, therefore, observed to perform better. Also, an improved performance is observed when both the users employ same codes $^{4}$. We reason that this behavior is dependent upon the code structure of the extended trellis, i.e., using the same component codes $\left((5 / 7)_{\text {octal }}\right.$ or $\left.(7 / 5)_{\text {octal }}\right)$ at both the users result in an extended trellis with better distance property when decoding of physical-layer network code is concerned (although, this may not be true in general).

We now proceed to the theoretical assessment of the joint decoding scheme using analytical approximations for the word-error rates. Since the joint decoding of the two messages according to (7) is computationally infeasible, we resort to iterative decoders such as those based on the turbo principle. The minimum distance decoding based on turbo codes can be approximated using the iterative decoding employing the well known BCJR algorithm. In the examples, we consider a turbo code with rate- $1 / 3$ and interleaver length $K=128$ $(N=384)$. Fig. 4 compares the corresponding analytical and simulation results where both users employ the same $(5 / 7)_{\text {octal }}$ constituent convolutional codes but with different power allocations to each of the transmitted symbols. We observe that the simulations are in excellent agreement with the bounds at high SNRs, which is as expected since the union bound becomes tight as SNR increases. For comparison purposes, we also provide the results when the two messages,

\footnotetext{
${ }^{4}$ Simulation results for different system configurations have been studied in detail in [25].
} 


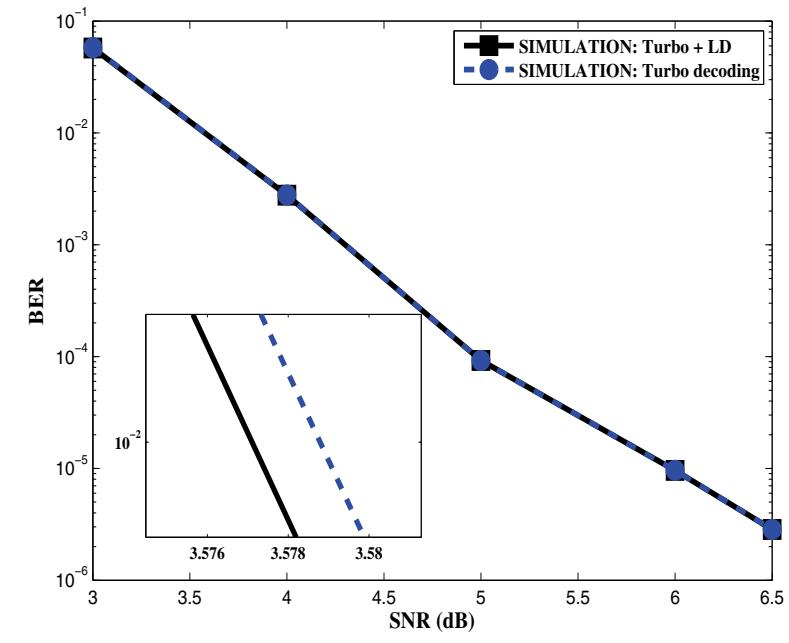

Fig. 3. Simulation results for turbo codes with different constituent encoders $\left((5 / 7)_{\text {octal }}\right.$ and $\left.(7 / 5)_{\text {octal }}\right)$ at the two source nodes with $N=384, P_{1}=1$ and $P_{2}=1$.

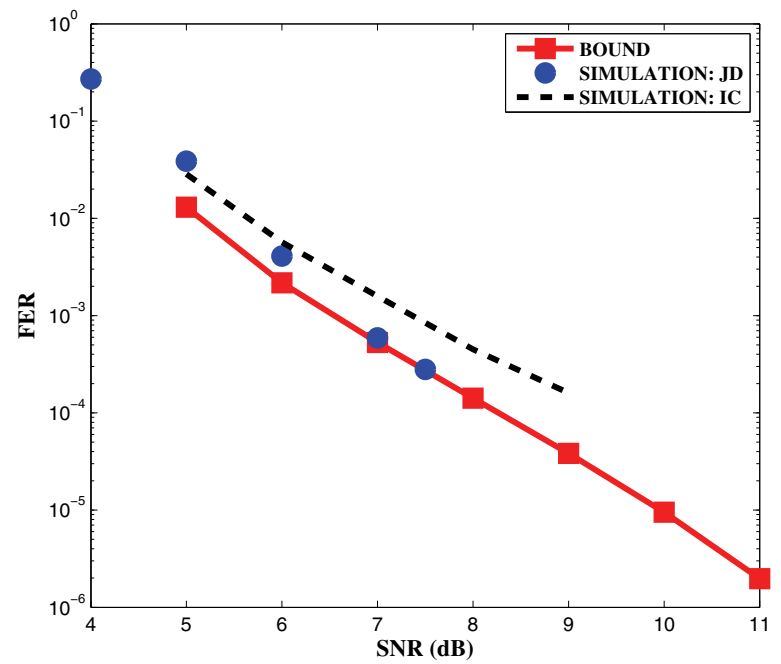

Fig. 4. Analytical approximations and simulation results for turbo codes with same $(5 / 7)_{\text {octal }}$ constituent encoders at the two source nodes with $N=384$, $P_{1}=1$ and $P_{2}=0.5$.

corresponding to each of the users, are decoded using successive interference cancellation at the relay. Since the power allocated at the transmitter of the first user is greater than that at the second user, the codeword of the first user is decoded in the first stage. The JD scheme clearly performs better and provides a gain of approximately $0.82 \mathrm{~dB}$ at a frame error rate of $10^{-3}$. In Fig. 5, results are provided when both the users employ different constituent encoders $\left((5 / 7)_{\text {octal }}\right.$ and $\left.(7 / 5)_{\text {octal }}\right)$ with same power allocation where similar observations are made.

In the above examples, we evaluate the bounds approximately using the weight enumerating function (WEF) of the individual codewords as shown in (15). In order to compute the bounds more accurately, it is required to compute the WEF using the extended trellis at the relay which can be evaluated using the techniques described in [26], [27]. Because of the

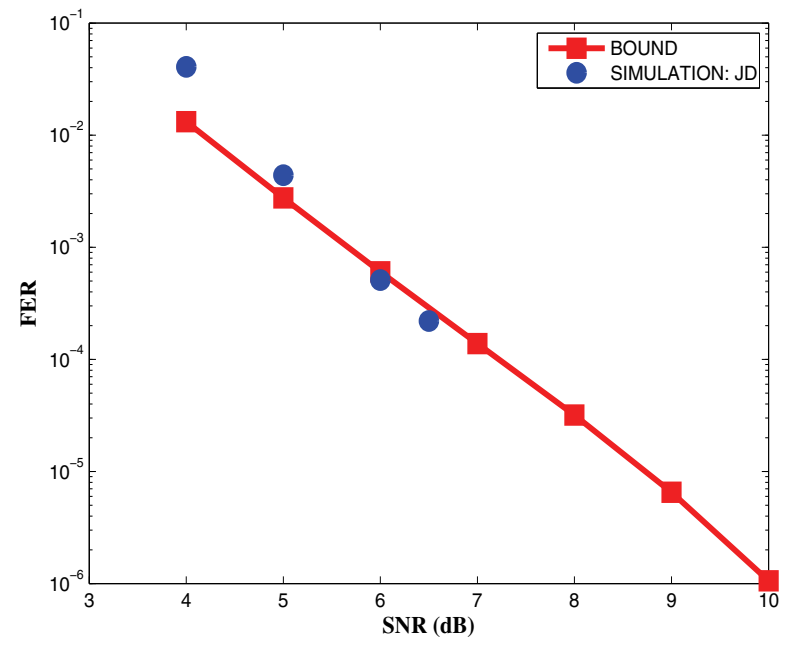

Fig. 5. Analytical approximations and simulation results for turbo codes with different constituent encoders $\left((5 / 7)_{\text {octal }}\right.$ and $\left.(7 / 5)_{\text {octal }}\right)$ at the two source nodes with $N=384, P_{1}=1$ and $P_{2}=1$.

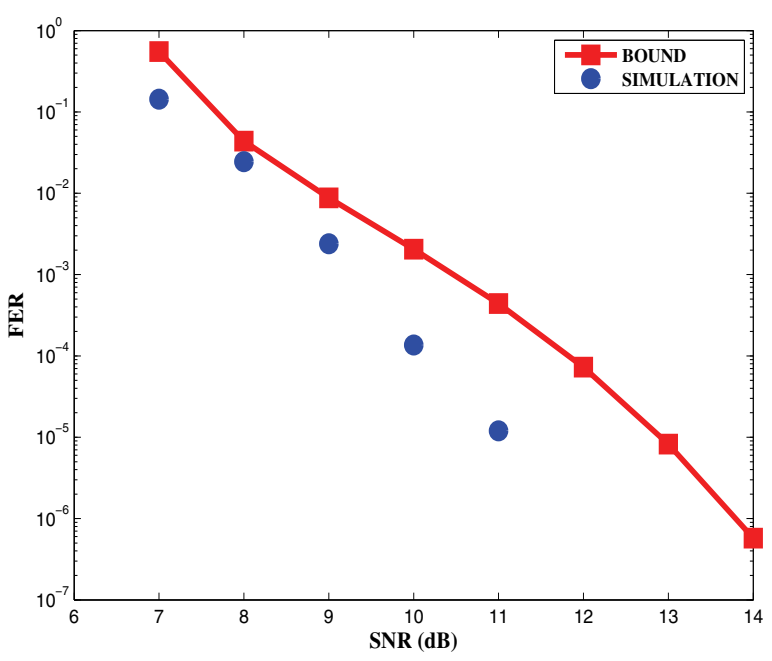

Fig. 6. Analytical approximations and simulation results with same $(5 / 7)_{\text {octal }}$ convolutional codes at the two source nodes with $N=256$, $P_{1}=1$ and $P_{2}=1$.

complexity involved due to the number of terms involved in order to track the distance spectra of the joint trellis codes, this computation is not practical, hence we do not include bound examples using this approach, because of which the computation of the bounds for convolutional codes (that use the Viterbi decoder) should be considered as approximations. Figs. 6 shows the performance results with rate- $1 / 2$ convolutional codes when the powers at the source nodes are the same with length $N=256$. The bounds computed for these codes are not tight since the WEF employed in the evaluation of the approximate bounds is not the true WEF.

We now provide results obtained from computer simulations for various decoding schemes discussed earlier when the channel is frequency selective. In our analysis, we assume that the code-rates at each of the nodes are the same $(R=1 / 2)$, and the length of the codewords are $N=256$. In the case 
TABLE I

SNR FOR BER OF $10^{-2}$ USING DIFFERENT DETECTION/DECODING SCHEMES.

\begin{tabular}{|c|c|c|}
\hline Decoding scheme & Fig. 7 & Fig. 8 \\
\hline LD - full states & $7.23 \mathrm{~dB}$ & $5.98 \mathrm{~dB}$ \\
VD - full states & $7.251 \mathrm{~dB}$ & $5.993 \mathrm{~dB}$ \\
LD - 64 states & $7.23 \mathrm{~dB}$ & $6.02 \mathrm{~dB}$ \\
VD - 64 states & $7.251 \mathrm{~dB}$ & $6.03 \mathrm{~dB}$ \\
LD - 16 states & $7.278 \mathrm{~dB}$ & $6.04 \mathrm{~dB}$ \\
VD - 16 states & $7.291 \mathrm{~dB}$ & $6.045 \mathrm{~dB}$ \\
LD - 4 states & 8.484 & $7.14 \mathrm{~dB}$ \\
VD - 4 states & 8.485 & $7.142 \mathrm{~dB}$ \\
MMSE scheme & $8.31 \mathrm{~dB}$ & $6.224 \mathrm{~dB}$ \\
\hline
\end{tabular}

of list decoding, the PLVA with a list size of $L=50$ is utilized. We start with an example of 3-tap ISI channels with real coefficients known at the relay for the entire duration of transmission of the codewords given as

$$
\begin{aligned}
& h_{1}(n)=\delta(n)+0.5 \delta(n-1)+0.1 \delta(n-2), \\
& h_{2}(n)=\delta(n)+0.3 \delta(n-1)+0.2 \delta(n-2) .
\end{aligned}
$$

Fig. 7 shows the performance when both the users allocate different power levels to the transmitted messages and employ different codes at each of the users $\left((5 / 7)_{\text {octal }}\right.$ and $\left.(7 / 5)_{\text {octal }}\right)$. By VD, we refer to the JCD/PNCD scheme implemented using a Viterbi decoder. We observe that the performance of the JCD/PNCD scheme closely matches the list decoding scheme, thereby implying its near-optimal performance (when the full trellis is considered). Results are also shown for lower complexity schemes implemented using the $M$-algorithm where only the best $M$ states during any instant of time are retained. Table I shows the comparison of the SNRs required with the list decoding and the JCD/PNCD schemes for different scenarios to achieve a bit error rate of $10^{-2}$.

Fig. 7 also show the performance obtained with the MMSE detection scheme. In our simulations we assume that $K_{1}=9$ and $K_{2}=5$ and consider only one iteration between the MMSE detector and the PNC decoder (i.e. no turbo equalization). The results illustrate that there is a considerable loss in the performance with the implementation of the MMSE scheme compared to the full-state Viterbi decoding. Table I further illustrates the comparison of MMSE schemes with the other decoding schemes.

We now provide examples with channels with impulse responses

$$
\begin{aligned}
h_{1}(n)= & (0.114+0.994 j) \delta(n)+(0.023+0.043 j) \delta(n-1) \\
& +(0.019+0.006 j) \delta(n-2), \\
h_{2}(n)= & (-0.67+0.75 j) \delta(n)+(0.12+0.16 j) \delta(n-1) \\
& +(0.028-0.006 j) \delta(n-2) .
\end{aligned}
$$

Fig. 8 shows the performance of different decoding schemes over frequency selective channels with 3-taps where we observe the similar behavior with the JCD/PNCD scheme performing close to the list decoding scheme (with full trellis). We observe that the performance of the JCD/PNCD scheme (marked as VD in the figure) closely matches the result of the list decoding scheme, thereby implying a near-optimal performance as in the previous example. Results with the reduced complexity $M$-algorithm as well as MMSE based linear

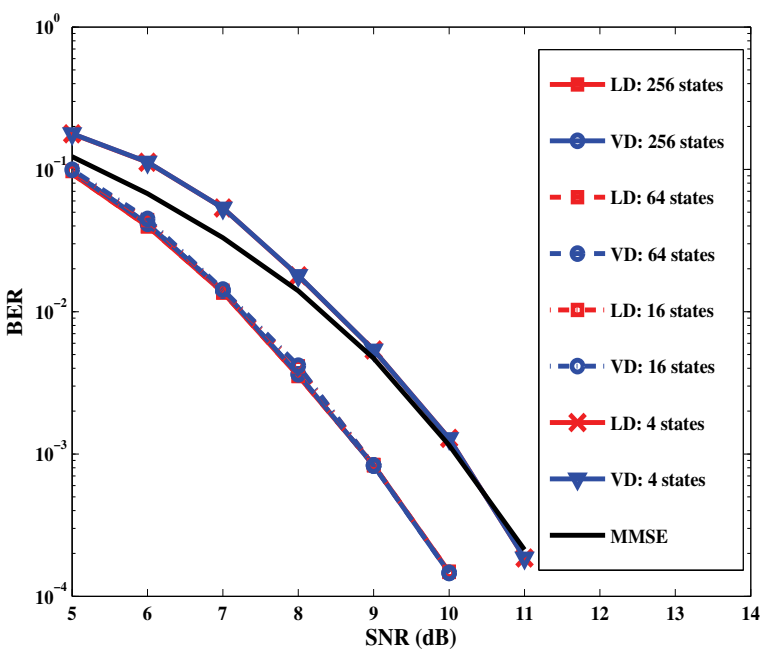

Fig. 7. Simulation results with different $\left((5 / 7)_{\text {octal }}\right.$ and $\left.(7 / 5)_{\text {octal }}\right)$ convolutional codes at the two source nodes over the 3-tap ISI channel example with $P_{1}=1$ and $P_{2}=0.5$.

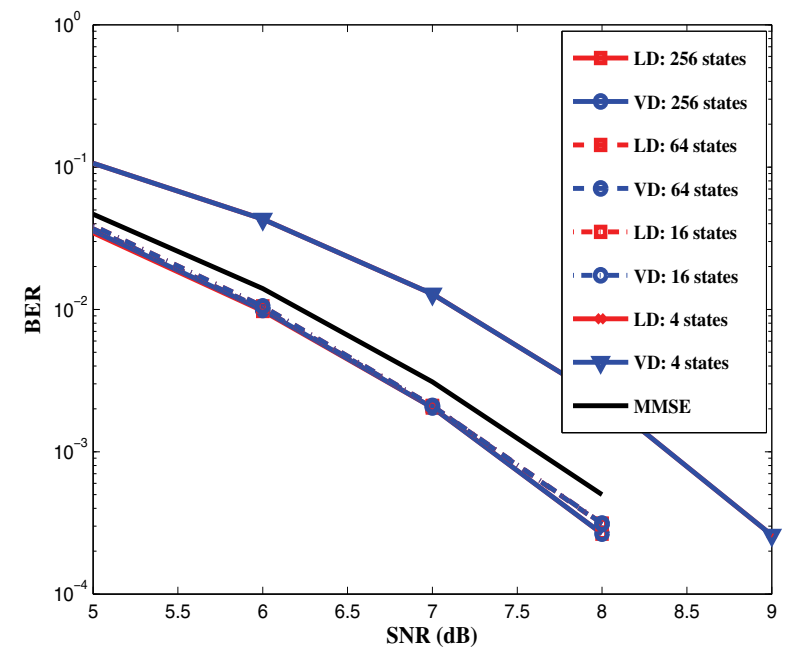

Fig. 8. Simulation results over 3-tap complex ISI channels in 28 with $P_{1}=1$ and $P_{2}=1$.

detection (with $K_{1}=9, K_{2}=5$ ) are also shown. As also observed in the previous example, there is a performance loss with the incorporation of the reduced complexity algorithms. For instance, in the case of MMSE based linear detection, in order to achieve a BER of $10^{-2}$, and additional $0.26 \mathrm{~dB}$ of SNR is required compared to the near-optimal JCD/PNCD scheme. Performance with lower complexity schemes using the $M$-algorithm are also shown and are listed in Table I.

We now study a two-way relay channel example suitable for an underwater acoustic communication channel. The multipath spread for these channels can be in the order of tens of $m s$ or more, hence for single carrier systems, leading to large number of channel taps [8]. For such cases, implementation of the JCD/PNCD scheme becomes infeasible for such cases and we resort to the suboptimal MMSE based decoding scheme. As a specific example, we consider channel example estimated from data recorded in a recent underwater acoustic communi- 


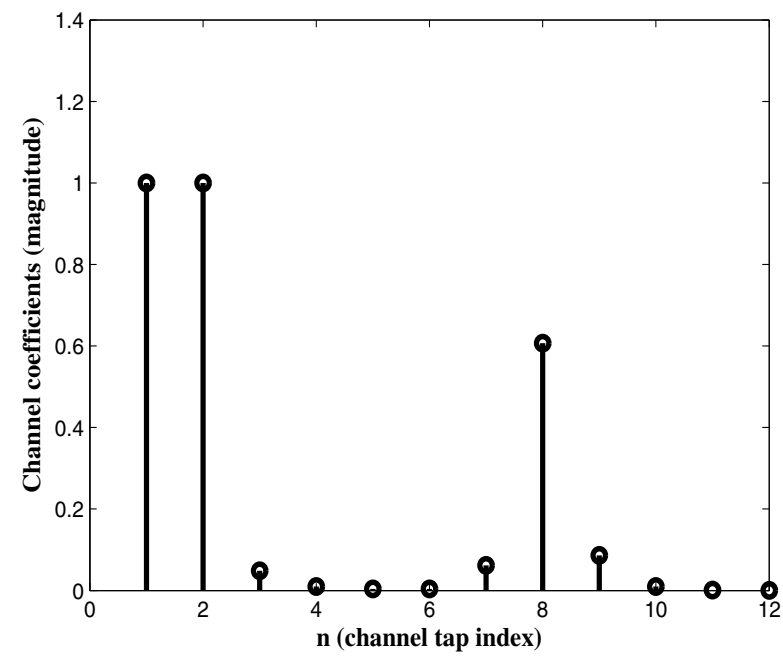

Fig. 9. Channel response for source 1 - relay link.

cation experiment, namely, mobile acoustic communications experiment 2010 (MACE 2010) [28]. Each user transmits its corresponding symbols over a bandwidth of $5 \mathrm{kHz}$ with a carrier frequency of $12.5 \mathrm{kHz}$. We choose two channels picked from different transmitter/receiver pairs and utilize them as the channels for the two sources communicating with the relay in our example. The delay spreads across the two sourcerelay links are $6.7 \mathrm{~ms}$ and $7.1 \mathrm{~ms}$, respectively. We assume that the symbol duration for both transmissions are $0.5 \mathrm{~ms}$, that results in the discrete channel model with 10 significant channel taps as shown in Figs. 9 and 10. The performance of the system over these channels with PNC for different MMSE filter lengths $K_{R}$ is shown in Fig. 11 (assuming same codes are employed at both the users). We observe that the performance of the system improves as the length of the filter increases. For instance, a performance gain of about $4 \mathrm{~dB}$ by incorporating an MMSE detector with filter length $K_{R}=15\left(K_{1}=9, K_{2}=5\right)$ as opposed to $K_{R}=9\left(K_{1}=5, K_{2}=3\right)$ for a BER of $10^{-2}$ is possible. We also show the results with $K_{R}=11$ $\left(K_{1}=5, K_{2}=5\right)$ in the same figure. The implementation of the JCD/PNCD scheme becomes infeasible for such channels (due to the large number of channel taps) as the number of trellis states involved are very large, and therefore, the results using this scheme are not included.

\section{CONCLUSIONS}

In this paper, we have investigated the decoding strategies at the relay for a two-way relay channel with physicallayer network coding, and proposed a way of approximately implementing the optimal decoding strategy utilizing a list decoding algorithm. We proposed a simplified joint decoding strategy, and via examples, demonstrated that for practical Gaussian noise channels, the list decoding performance can be well approximated using this scheme. Using this JD scheme, we derived an analytical upper bound of the word-error probability using the union bound based on the JD scheme. We further developed decoding strategies at the relay to decode the network coded sequence for a two-way relay channel with

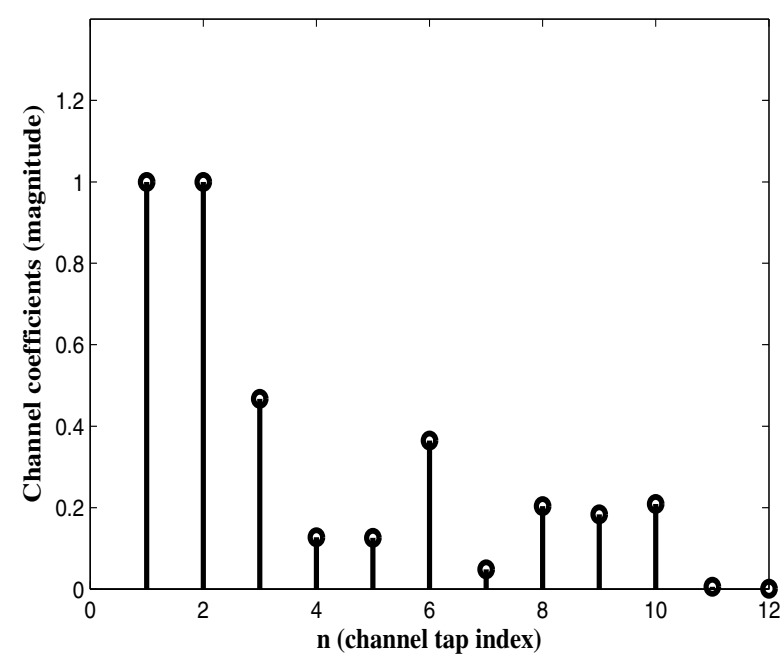

Fig. 10. Channel response for source 2 - relay link.

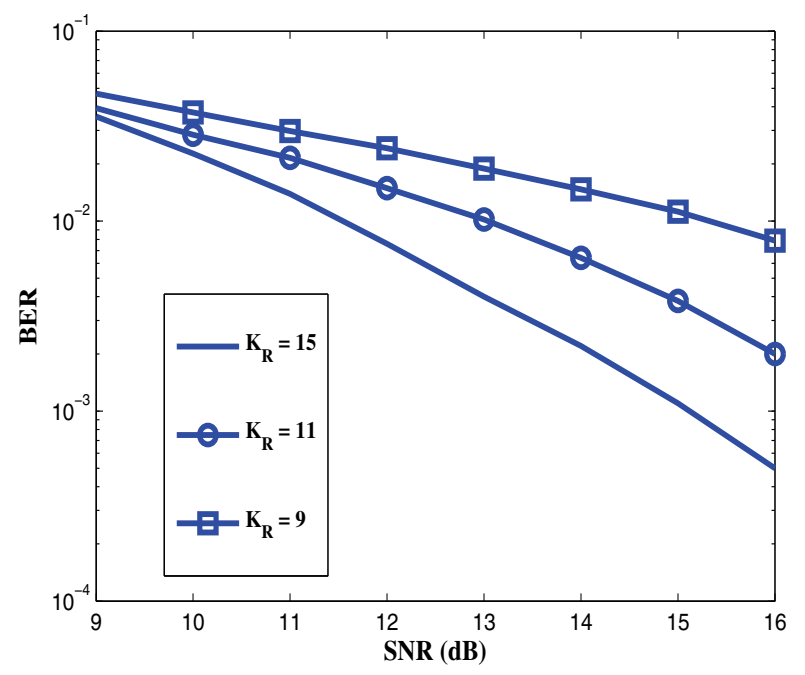

Fig. 11. Performance of MMSE detector using different filter lengths with $P_{1}=1$ and $P_{2}=1$ over the 10-tap ISI channels (example taken from the MACE 2010).

inter-symbol interference. We showed using numerical examples that a joint channel detector/physical layer network coded sequence decoder performs equally well and due to the reduced complexity as compared to the LD scheme, can instead be incorporated. We further investigated a low complexity channel detector using a linear detection scheme, where the MMSE equalizer generates soft information corresponding to the superimposed symbols and passes it to the PNC decoder. The simulation results are provided showing the bit error rate performances of the different schemes and to demonstrate the decoding results and the analytical approximations.

\section{REFERENCES}

[1] R. Ahlswede, N. Cai, S.-Y. Li, and R. Yeung, "Network information flow," IEEE Trans. Inf. Theory, vol. 46, no. 4, pp. 1204-1216, July 2000.

[2] S. Zhang, S.-C. Liew, and P. P. Lam, "Physical-layer network coding," in 2006 ACM Mobicom. 
[3] P. Popovski and H. Yomo, "Physical network coding in two-way wireless relay channels," in Proc. 2007 IEEE Int. Conf. on Comm., pp. 707-712.

[4] S. Zhang and S.-C. Liew, "Channel coding and decoding in a relay system operated with physical-layer network coding," IEEE J. Sel. Areas Commun., vol. 27, no. 5, pp. 788-796, June 2009.

[5] T. Koike-Akino, P. Popovski, and V. Tarokh, "Optimized constellations for two-way wireless relaying with physical network coding," IEEE $J$. Sel. Areas Commun., vol. 27, no. 5, pp. 773-787, June 2009.

[6] D. Wübben and Y. Lang, "Generalized joint channel coding and physical network coding for two-way relay systems," in Proc. 2010 IEEE Vehicular Tech. Conf. - Spring, pp. 1-5.

[7] D. To and J. Choi, "Convolutional codes in two-way relay networks with physical-layer network coding," IEEE Trans. Wireless Commun., vol. 9, no. 9, pp. 2724-2729, Sep. 2010.

[8] M. Stojanovic, "Underwater acoustic communications: design considerations on the physical layer," in Proc. Wireless on Demand Net. Sys. and Services, pp. 1-10.

[9] H. Gacanin and F. Adachi, "Performance of physical layer network coding in a frequency-selective fading channel," in Proc. 2009 IEEE Int. Symp. on Personal, Indoor and Mobile Radio Comm., pp. 928-932.

[10] T. Koike-Akino, P. Popovski, and V. Tarokh, "Two-way relaying with network coding for frequency-selective fading channels," in Proc. 2008 Asilomar Conf. on Sig., Sys. and Comp., pp. 2221-2225.

[11] M. Ju and I.-M. Kim, "Error performance analysis of BPSK modulation in physical-layer network-coded bidirectional relay networks," IEEE Trans. Commun., vol. 58, no. 10, pp. 2770-2775, Oct. 2010.

[12] T. Yang, I. Land, T. Huang, J. Yuan, and Z. Chen, "Distance properties and performance of physical layer network coding with binary linear codes for Gaussian two-way relay channels," in Proc. 2011 IEEE Int. Symp. on Inform. Theory, pp. 2070-2074.

[13] J. Hu and T. M. Duman, "Cooperation over frequency-selective fading relay channels," IEEE Trans. Wireless Commun., vol. 7, no. 12, pp. 5072-5081, Dec. 2008.

[14] M. Tuchler, R. Koetter, and A. Singer, "Turbo equalization: principles and new results," IEEE Trans. Commun., vol. 50, no. 5, pp. 754-767, May 2002

[15] U. Bhat and T. M. Duman, "Decoding strategies for physical-layer network coding over frequency selective channels," in Proc. 2012 IEEE Wireless Comm. and Netw. Conf., pp. 12-17.

[16] N. Seshadri and C.-E. Sundberg, "List Viterbi decoding algorithms with applications," IEEE Trans. Commun., vol. 42, no. 234, pp. 313-323, Feb./Mar./Apr. 1994.

[17] C. Nill and C.-E. Sundberg, "List and soft symbol output Viterbi algorithms: extensions and comparisons," IEEE Trans. Commun., vol. 43, no. 234, pp. 277-287, Feb./Mar./Apr. 1995.

[18] K. Narayanan and G. Stuber, "List decoding of turbo codes," IEEE Trans. Commun., vol. 46, no. 6, pp. 754-762, June 1998.

[19] S. Benedetto and G. Montorsi, "Unveiling turbo codes: some results on parallel concatenated coding schemes," IEEE Trans. Inf. Theory, vol. 42, no. 2, pp. 409-428, Mar. 1996.

[20] T. M. Duman and M. Salehi, "Performance bounds for turbo-coded modulation systems," IEEE Trans. Commun., vol. 47, no. 4, pp. 511521, Apr. 1999

[21] U. Bhat, D. Fertonani, and T. M. Duman, "Approximate performance analysis for linear codes in superposition schemes over Gaussian broadcast channels," IEEE Trans. Commun., vol. 58, no. 8, pp. 2177-2182, Aug. 2010.

[22] N. Seshadri and C.-W. Sundberg, "Generalized Viterbi algorithms for error detection with convolutional codes," in Proc. 1989 IEEE Global Telecom. Conf., pp. 1534-1538.

[23] J. Anderson and S. Mohan, "Sequential coding algorithms: a survey and cost analysis," IEEE Trans. Commun., vol. 32, no. 2, pp. 169-176, Feb. 1984.
[24] S. Simmons, "Breadth-first trellis decoding with adaptive effort," IEEE Trans. Commun., vol. 38, no. 1, pp. 3-12, Jan. 1990.

[25] U. Bhat, "Practical coding schemes for multi-user communications," Ph.D. dissertation, Arizona State University, Oct. 2011.

[26] D. Divsalar, S. D. and F. Pollara, and R. J. McEliece, "Transfer function bounds on the performance of turbo codes," TDA Progress Rep. 42-122, JPL, Tech. Rep., Aug. 1995.

[27] T. M. Duman, "Turbo codes and turbo coded modulation systems: analysis and performance bounds," Ph.D. dissertation, Northeastern University, May 1998.

[28] K. Davidson, "MACE10 - mobile acoustic communications experiment 2010 - Quick-Look Cruise report," Tech. Rep., Aug. 2010.

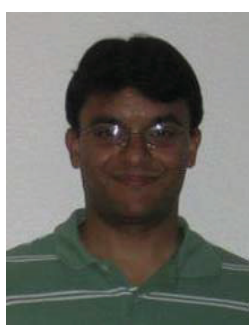

Uttam Bhat received his Ph.D. degree in Electrical Engineering from Arizona State University in October 2011. He received his B.E. degree in Electronics and Communications from Sri Jayachamarajendra College of Engineering, Mysore, India in 2004, and M.S. degree in Electrical Engineering from Arizona State University in May 2008.

$\mathrm{He}$ currently holds the position as Member of Technical Staff at EnVerv Inc., Milpitas CA, where his primary responsibility is in design of physical layer solutions for power line communications. He has previously held positions at EmSyS, Larsen and Toubro Ltd., India and Mindspeed Technologies Inc., Newport Beach CA. His areas of interests are in practical channel coding schemes, power line communications, multi-user communications, detection/decoding schemes for high-speed communication systems, and signal processing for wireless/digital communications.

Dr. Bhat has co-authored several IEEE conference and journal papers. $\mathrm{He}$ has been listed as exemplary reviewer for IEEE COMMUNICATIONS LETTERS in 2012. He has served as reviewer for several IEEE magazines, journals and conferences as well.

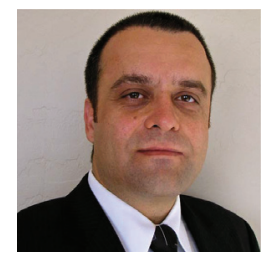

Tolga M. Duman (S'95-M'98-SM'03-F'11): Tolga M. Duman is a Professor at the Electrical and Electronics Engineering Department of Bilkent University in Turkey, and is on leave from the School of Electrical, Computer and Energy Engineering of Arizona State University. He received the B.S. degree from Bilkent University in Turkey in 1993, M.S. and Ph.D. degrees from Northeastern University, Boston, in 1995 and 1998, respectively, all in electrical engineering. Prior to joining Bilkent University in September 2012, he has been with the Electrical Engineering Department of Arizona State University first as an Assistant Professor (1998-2004), then as an Associate Professor (2004-2008), and starting August 2008 as a Professor. Dr. Duman's current research interests are in systems, with particular focus on communication and signal processing, including wireless and mobile communications, coding/modulation, coding forwireless communications, data storage systems and underwater acoustic communications.

Dr. Duman is a Fellow of IEEE, a recipient of the National Science Foundation CAREER Award and IEEE Third Millennium medal. His publications include a book on MIMO Communications (by Wiley in 2007), over 50 journal papers and over 100 conference papers. He served as an editor for IEEE TRANSACTIONS ON WIRELESS COMMUNICATIONS (200308), IEEE TRANSACTIONS ON COMMUNiCATIONS (2007-2012) and IEEE COMMUNICATIONS SURVEYS AND TUTORIALS (2002-07). He is currently the coding and communication theory area editor for IEEE TRANSACTIONS ON COMMUniCATIONS (2011-present) and an editor for Elsevier Physical Communications Journal (2010-present). 